\title{
Associations of high-mobility group box 1 and receptor for advanced glycation end products with acute lung injury in patient with acute aortic dissection
}

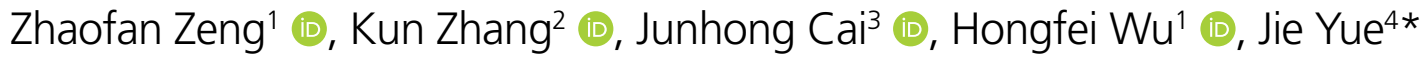

\section{SUMMARY}

OBJECTIVE: To investigate the associations of high-mobility group box 1 and its specific receptor, receptor for advanced glycation end products with acute lung injury in patients with acute aortic dissection.

METHODS: A total of 96 acute aortic dissection patients were divided into acute aortic dissection with acute lung injury group (38 cases) and acute aortic dissection without acute lung injury group (58 cases), according to partial pressure of oxygen/fraction of inspired oxygen. In addition, 44 healthy individuals were selected for the control group. The blood samples were taken. The serum high-mobility group box 1 and receptor for advanced glycation end products levels were detected by enzyme-linked immunosorbent assay, and the partial pressure of oxygen/fraction of inspired oxygen was measured.

RESULTS: $24 \mathrm{~h}$ after admission, the high-mobility group box 1 and receptor for advanced glycation end products levels in acute aortic dissection with acute lung injury and acute aortic dissection without acute lung injury groups were significantly higher than those in the control group, respectively $(p<0.05)$, and each index in acute aortic dissection with acute lung injury group was significantly higher than that in acute aortic dissection without acute lung injury group $(\mathrm{p}<0.05)$. At each time point within $96 \mathrm{~h}$ after admission, compared with acute aortic dissection without acute lung injury group, in acute aortic dissection with acute lung injury group, the high-mobility group box 1 and receptor for advanced glycation end products levels were increased, respectively, and the partial pressure of oxygen/ fraction of inspired oxygen was decreased. The correlation analysis showed that, in acute aortic dissection patients, the high-mobility group box 1 and receptor for advanced glycation end products levels were negatively correlated with partial pressure of oxygen/fraction of inspired oxygen, respectively $(p<0.05)$.

CONCLUSIONS: The serum high-mobility group box 1 and receptor for advanced glycation end products levels may be associated with the occurrence of acute lung injury in acute aortic dissection patients. Monitoring the high-mobility group box 1 and receptor for advanced glycation end products levels can evaluate the risk of acute aortic dissection with acute lung injury .

KEYWORDS: Aortic dissection. Acute lung injury. High-mobility group box 1. Receptor for advanced glycation end products.

${ }^{1}$ Hainan General Hospital, Hainan Affiliated Hospital of Hainan Medical University, Department of Vascular Surgery - Haikou, China.

${ }^{2}$ Hainan General Hospital, Hainan Affiliated Hospital of Hainan Medical University, Medical Care Center, Department of Geriatrics - Haikou, China. ${ }^{3}$ Hainan General Hospital, Hainan Affiliated Hospital of Hainan Medical University, Molecular Laboratory Center - Haikou, China.

${ }^{4}$ The First Affiliated Hospital of Guangzhou Medical University, Department of Cardiovascular Surgery - Guangzhou, China.

*Corresponding author: yuejiegz2@126.com

Conflicts of interest: the authors declare there are no conflicts of interest. Funding: Natural Science Foundation of Hainan Province (818MS128) and Hainan Province Clinical Medical Center.

Received on June 07, 2021. Accepted on July 20, 2021. 


\section{INTRODUCTION}

Acute aortic dissection (AAD) is a serious cardiovascular disease. In $\mathrm{AAD}$, the blood flows into the cystic-degenerated middle aortic layer through the avulsion of aortic intima to form a dissection hematoma that expands in the aorta driven by blood pressure ${ }^{1}$. Acute lung injury (ALI) is one of the common complications of AAD. It is characterized by diffuse alveolar epithelial cell injury and increased capillary permeability. The incidence of AAD complicated ALI is more than $50 \%^{2}$. AAD with ALI can prolong the time of ventilator use, prolong the length of hospital stay, increase the treatment cost, increase the mortality, and seriously affect the prognosis of patients ${ }^{3,4}$. Therefore, it is of great significance to study the pathogenesis of AAD with ALI for improving the prognosis of patients. The mechanism of AAD with ALI has not been fully elucidated. It is well known that the changes in cellular and biochemical regulatory pathways eventually lead to the AAD with ALI. These changes include the increased inflammatory response and oxidative stress, and matrix metalloproteinases ${ }^{5,6}$.

High-mobility group box 1 (HMGB1) is a member of HMGB family. It is located on human chromosome 13q12 and consists of 215 amino acid residues ${ }^{7}$. As a nuclear nonhistone protein, HMGB1 has a strong pro-inflammatory effect and plays a key role in the initiation and maintenance of inflammatory cascade reaction ${ }^{8}$. When the body is in a steady state without external stimulation, HMGB1 mainly exists in the nucleus. Under stress condition, HMGB1 is secreted outside the cell through nonclassical or passive release pathway. It interacts with the specific receptor, receptor for advanced glycation end products (RAGE), to activate the mitogen-activated protein kinase pathway and nuclear factor kappa $B$, thus inducing the secretion of tumor necrosis factor $\alpha$, interleukin $1 \beta$, interleukin 6 , and other inflammatory factors that produce the inflammatory cascade reaction'. Therefore, the HMGB1 and RAGE may be related to AAD with ALI. This study monitored the expression levels of HMGB1 and RAGE in AAD patients complicated with ALI, analyzed the associations of HMGB1 and RAGE with oxygenation index (partial pressure of oxygen/fraction of inspired oxygen, $\mathrm{PaO}_{2} / \mathrm{FiO}_{2}$ ), and explore their clinical significances.

\section{SUBJECTS AND METHODS}

\section{Subjects}

A total of $96 \mathrm{AAD}$ patients treated in our hospital from January 2018 to June 2020 were enrolled in this study. There were 58 males and 38 females. The age of patients was $33-82$ years old, with average age of $76.32 \pm 10.34$ years old.
There were 59, 30, 78, and 32 cases with smoking history, diabetes history, hypertension history, and coronary heart disease history, respectively. The erythrocyte sedimentation rate was $4.47 \pm 1.27 \mathrm{~mm} / \mathrm{h}$. The serum creatinine level was $90.05 \pm 22.19 \mathrm{nmol} / \mathrm{mL}$. The serum brain natriuretic peptide level was $102.05 \pm 25.24 \mathrm{pg} / \mathrm{mL}$. The prothrombin time was $13.99 \pm 1.52 \mathrm{~s}$ and the ejection fraction was $64.12 \pm 11.05 \%$. According to the $\mathrm{PaO}_{2} / \mathrm{FiO}_{2}$ under static oxygen inhalation, the patients were divided into AAD with ALI group (38 cases) and AAD without ALI group ( 58 cases). In addition, 44 healthy individuals receiving physical examination were randomly selected as the control group. This study was approved by the ethics committee of Hainan General Hospital. The signed informed consent was obtained from all subjects.

\section{Inclusion and exclusion criteria}

The inclusion criteria were as follows:

1. the AAD was confirmed by computed tomography angiography or digital subtraction angiograph;

2. the patients were admitted within $24 \mathrm{~h}$ after AAD onset; and

3. the patients received the conservative treatment for more than 4 days after admission.

The exclusion criteria were as follows:

1. the patients were admitted after $24 \mathrm{~h}$ from AAD onset;

2. the patients received surgery (interventional therapy) or died within 4 days after admission;

3. the patients had malignant tumor or immune diseases;

4. the patients had taken anti-inflammatory drugs or immunosuppressants; and

5. the patients had the respiratory disease history.

\section{Study method}

After admission, the patients were immediately admitted to the intensive care unit, and were given oxygen inhalation, electrocardiogram monitoring, analgesia, antihypertension, defecation, and other symptomatic treatment. The mechanical ventilation was performed, if necessary. For all patients, the arterial blood was drawn every $4 \mathrm{~h}$ after admission. The $\mathrm{PaO}_{2} / \mathrm{FiO}_{2}$ was measured. At the corresponding time point, the elbow venous blood was drawn. The blood was centrifuged at $3000 \mathrm{r} / \mathrm{min}$ for $15 \mathrm{~min}$. The supernatant was obtained and stored at $-80^{\circ} \mathrm{C}$ in a refrigerator for testing. The levels of HMGB1 and RAGE were detected by enzyme-linked immunosorbent assay. The detection operations were in strict accordance with the instruction of kits. In the control group, the morning fasting elbow venous blood was drawn only once. The detection operations of HMGB1 and RAGE were the same with those in AAD patients. 


\section{Statistical analysis}

All statistical analysis was carried out using SPSS 20.0 software (SPSS Inc., Chicago, IL, USA). The enumeration data were presented as number and rate, and the comparison between AAD with ALI and AAD without ALI groups was performed using $\chi^{2}$ test. The measurement data were expressed as the mean \pm standard deviation. The comparison between AAD with ALI and AAD without ALI groups was performed using t-test. The comparison among AAD with ALI, AAD without ALI, and the control groups was performed using analysis of variance. The correlations of HMGB1, RAGE, and $\mathrm{PaO}_{2} / \mathrm{FiO}_{2}$ were analyzed by Spearman's rank correlation analysis. $\mathrm{P}<0.05$ was considered as statistically significant.

\section{RESULTS}

\section{General information of acute aortic dissection patients}

General information of AAD patients was shown in Table 1. There was no significant difference of gender, age, smoking history, diabetes history, hypertension history, coronary heart disease history, erythrocyte sedimentation rate, serum creatinine level, serum brain natriuretic peptide level, prothrombin time, or ejection fraction between AAD with ALI group and AAD without ALI group ( $>0.05)$.
Comparison of high-mobility group box 1 and receptor for advanced glycation end products levels among acute aortic dissection with acute lung injury, acute aortic dissection without acute lung injury, and control groups

As shown in Table 2, the levels of HMGB1 and RAGE at $24 \mathrm{~h}$ after admission in AAD with ALI and AAD without ALI groups were significantly higher than those in the control group, respectively $(\mathrm{p}<0.05)$. Compared with AAD without ALI group, the levels of HMGB1 and RAGE in AAD with ALI group were significantly increased, respectively $(\mathrm{p}<0.05)$.

Table 2. Comparison of high-mobility group box 1 and receptor for advanced glycation end products levels among acute aortic dissection with acute lung injury, acute aortic dissection without acute lung injury, and the control groups.

\begin{tabular}{l|c|c|c} 
Group & $n$ & HMGB1 $(\mathrm{ng} / \mathrm{mL})$ & RAGE $(\mu \mathrm{g} / \mathrm{L})$ \\
\hline AAD with ALI & 38 & $89.21 \pm 18.21^{\mathrm{ab}}$ & $105.21 \pm 20.87^{\mathrm{ab}}$ \\
\hline $\begin{array}{l}\text { AAD without } \\
\text { ALI }\end{array}$ & 58 & $48.24 \pm 7.05^{\mathrm{a}}$ & $73.75 \pm 12.33^{\mathrm{a}}$ \\
\hline Control & 44 & $12.95 \pm 3.29$ & $22.78 \pm 4.40$ \\
\hline $\mathrm{F}$ & & 521.770 & 385.336 \\
\hline $\mathrm{t}$ & & $<0.001$ & $<0.001$ \\
\hline
\end{tabular}

AAD: acute aortic dissection; ALI: acute lung injury; HMGB1: high-mobility group box 1; RAGE: receptor for advanced glycation end products. ${ }^{a} p<0.05$ compared with the control group. ${ }^{b} p<0.05$ compared with AAD without ALI group.

Table 1. General information of acute aortic dissection patients.

\begin{tabular}{l|c|c|c|c} 
Group & AAD with ALI & AAD without ALI & t/ $\chi^{2}$ & $P$ \\
\cline { 1 - 3 } $\mathrm{n}$ & 38 & 58 & 0.167 & 0.683 \\
\hline Gender, $\mathrm{n}(\%)$ & & & & \\
\hline Male & $22(57.89)$ & $36(62.07)$ & & \\
\hline Female & $16(42.11)$ & $22(37.93)$ & & 0.117 \\
\hline Sme (years) & $77.58 \pm 8.05$ & $75.04 \pm 7.44$ & 1.583 & 0.879 \\
\hline Diabetes history, $\mathrm{n}(\%)$ & $23(60.53)$ & $36(62.07)$ & 0.023 & 0.955 \\
\hline Hypertension history, $\mathrm{n}(\%)$ & $12(31.58)$ & $18(31.03)$ & 0.003 & 0.640 \\
\hline CHD history, $\mathrm{n}(\%)$ & $30(78.95)$ & $48(82.76)$ & 0.219 & 0.883 \\
\hline ESR (mm/h) & $13(34.21)$ & $19(32.76)$ & 0.022 & 0.081 \\
\hline Creatinine $(\mathrm{nmol} / \mathrm{mL})$ & $4.33 \pm 1.04$ & $4.70 \pm 0.98$ & 1.766 & 0.289 \\
\hline BNP $(\mathrm{pg} / \mathrm{mL})$ & $92.23 \pm 20.04$ & $88.06 \pm 17.83$ & 1.067 & 0.358 \\
\hline Prothrombin time $(\mathrm{s})$ & $103.27 \pm 23.04$ & $99.36 \pm 18.27$ & 0.924 & 0.079 \\
\hline Ejection fraction $(\%)$ & $14.16 \pm 1.05$ & $13.67 \pm 1.23$ & 1.777 & 0.449 \\
\hline
\end{tabular}

AAD: acute aortic dissection; ALI: acute lung injury; CHD: coronary heart disease; ESR: erythrocyte sedimentation rate; BNP: brain natriuretic peptide. 
Changes of high-mobility group box 1 , receptor for advanced glycation end products, and partial pressure of oxygen/ fraction of inspired oxygen in acute aortic dissection patients within $96 \mathrm{~h}$ after admission

The changes of HMGB1, RAGE, and $\mathrm{PaO}_{2} / \mathrm{FiO}_{2}$ in AAD patients within $96 \mathrm{~h}$ after admission were shown in Figure 1. After admission, in both AAD with ALI and AAD without ALI groups, the HMGB1 and RAGE levels increased gradually, and the $\mathrm{PaO}_{2} / \mathrm{FiO}_{2}$ decreased gradually. With the extension of time, the HMGB1 and RAGE levels reached the highest value, followed by gradually decreasing, and the $\mathrm{PaO}_{2} / \mathrm{FiO}_{2}$ reached the lowest value, followed by gradually increasing. At each time point, compared with AAD without ALI group, in AAD with ALI group, the HMGB1 and RAGE levels were increased, respectively, and the $\mathrm{PaO}_{2} / \mathrm{FiO}_{2}$ was decreased (Figure 1).

\section{Correlations of high-mobility group} box 1 , receptor for advanced glycation end products, and partial pressure of oxygen/fraction of inspired oxygen in acute aortic dissection patients

The Spearman rank correlation analysis showed that, in $96 \mathrm{AAD}$ patients, the HMGB1 level was negatively correlated with $\mathrm{PaO}_{2} / \mathrm{FiO}_{2}(\mathrm{r}=-0.978, \mathrm{p}<0.001)$. The RAGE level was negatively correlated with $\mathrm{PaO}_{2} / \mathrm{FiO}_{2}(\mathrm{r}=-0.944, \mathrm{p}<0.001)$. The HMGB1 level was positively correlated with RAGE $(\mathrm{r}=0.978, \mathrm{p}<0.001)$.

\section{DISCUSSION}

The pathogenesis of AAD with ALI has not been fully clarified, and it is closely related to the excessive inflammatory cascade reaction. When AAD occurs, the aortic intima is avulsed. The blood flows into the aortic false lumen and contact with the extracellular matrix of aortic media. This leads to the activation of inflammatory cells and release of a large number of inflammatory mediators and pro-inflammatory factors, which then mediate the waterfall-like inflammatory cascade reaction ${ }^{10,11}$. There are extensive capillary beds in lung tissue, where the inflammatory cells gather. In ADD, these inflammatory cells are activated in the lung tissue. They release the inflammatory mediators that damage the pulmonary capillary endothelial cells. This leads to the increased microvascular permeability, decreased alveolar compliance, increased intrapulmonary shunt, and intractable hypoxemia ${ }^{12}$. Therefore, the oxygenation index $\mathrm{PaO}_{2} / \mathrm{FiO}_{2}$ is an important indicator of AAD with ALI.

HMGB1/RAGE signaling pathway exists in the constituent cells of various respiratory systems, and plays an important role in the pathogenesis of various respiratory diseases. Under stress condition, HMGB1 is released from the nucleus to the cytoplasm and further the extracellular matrix. It is internalized to the lysosome through the RAGE-mediated endocytosis, further transferring and activating the appropriate activation pathways ${ }^{13,14}$. It is suggested that the intervention of HMGB1/RAGE signaling pathway can inhibit the inflammatory response ${ }^{15}$. Zhou et al. ${ }^{16}$ have found that inhibition of long noncoding RNA nuclear paraspeckle assembly transcript 1 can inhibit the activation of HMGB1/RAGE signaling pathway, thus antagonizing the lipopolysaccharide-induced acute injury and inflammatory response of alveolar epithelial cells. In our study, $24 \mathrm{~h}$
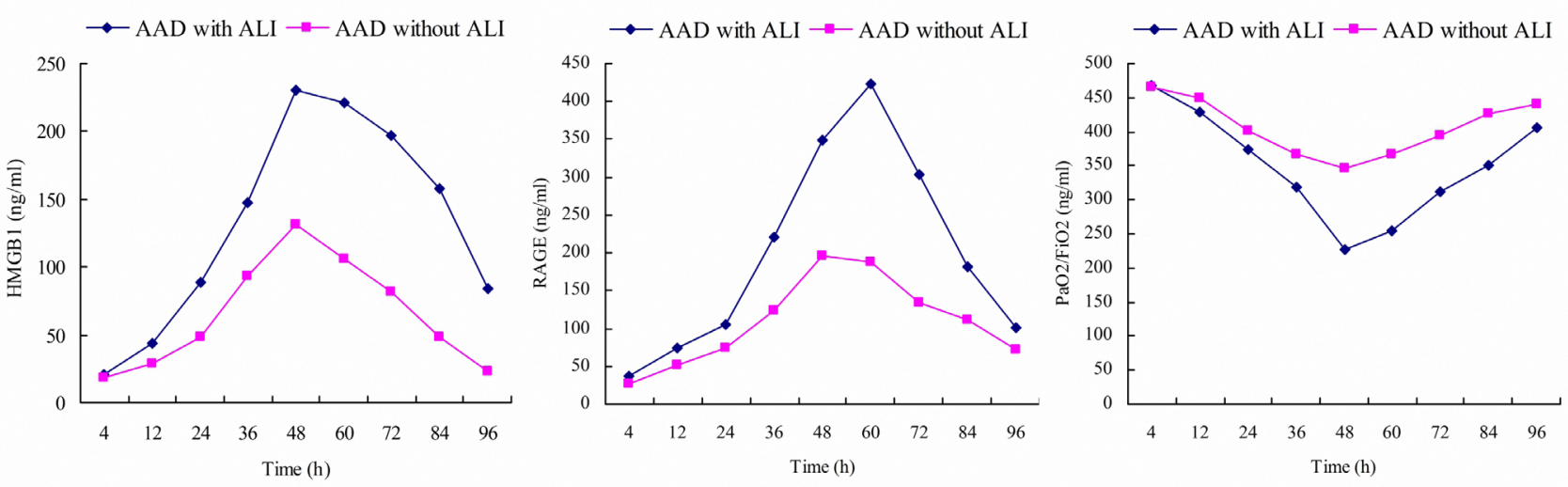

AAD: acute aortic dissection; ALI: acute lung injury; HMGB1: high-mobility group box 1; RAGE: receptor for advanced glycation end products; PaO $/$ $\mathrm{FiO}_{2}$ : partial pressure of oxygen/fraction of inspired oxygen.

Figure 1. Changes of high-mobility group box 1, receptor for advanced glycation end products, and partial pressure of oxygen/fraction of inspired oxygen in acute aortic dissection patients within $96 \mathrm{~h}$ after admission. 
after admission, the HMGB1 and RAGE levels in AAD with ALI and AAD without ALI groups were significantly higher than those in the control group. This indicates that, whether with or without ALI, the HMGB1 and RAGE levels in AAD patients are higher than those in healthy people.

In this study, within $96 \mathrm{~h}$ after admission, in both AAD with ALI and AAD without ALI groups, the HMGB1 and RAGE levels increased gradually, followed by decreasing, and the $\mathrm{PaO}_{2} / \mathrm{FiO}_{2}$ decreased gradually, followed by increasing. At each time point within $96 \mathrm{~h}$ after admission, compared with AAD without ALI group, in AAD with ALI group, the HMGB1 and RAGE levels were increased, and the $\mathrm{PaO}_{2} / \mathrm{FiO}_{2}$ was decreased. The correlation analysis showed that, in AAD patients, HMGB1 and RAGE levels were negatively correlated with $\mathrm{PaO}_{2} / \mathrm{FiO}_{2}$, respectively. This suggests that the activation of HMGB1/RAGE signaling pathway may be the key factor of ALI in AAD patients. The higher the HMGB1 and RAGE levels are, the more severe the inflammatory reaction is, and the more severe the lung injury is. With the decrease of HMGB1 and RAGE levels, the inflammatory reaction is decreased, and the lung injury is mitigated. They are closely related.

In conclusion, the serum HMGB1 and RAGE levels may be associated with the occurrence of ALI in AAD patients. The HMGB1/RAGE signaling pathway may play an important role in AAD with ALI. With the increase of HMGB1 and RAGE levels, the degree of ALI is gradually aggravated. Monitoring the HMGB1 and RAGE levels can evaluate the risk of AAD with ALI, which plays a good warning role in clinical practice. In-depth study of HMGB1/RAGE signaling pathway may provide potential therapeutic target for $\mathrm{AAD}$ patients with ALI. The limitation of this study is that the sample size is relatively small which may affect the results. In our subsequent studies, the sample size should be enlarged further for obtaining more convincing outcomes.

\section{AUTHORS' CONTRIBUTIONS}

JY: Conceptualization, Data curation, Formal analysis. ZZ: Funding acquisition, Investigation. KZ: Methodology, Project administration. JC: Supervision, Validation, Visualization. HW: Writing-original draft.

\section{REFERENCES}

1. Modi A, Diprose P, Tsang G. Complete avulsion of right coronary artery caused by acute type-A aortic dissection. Heart. 2013;99(21):1628. https://doi.org/ 10.1136/ heartjnl-2013-304066.

2. Wu Z, Ruan Y, Chang J, Li B, Ren W. Angiotensin II is related to the acute aortic dissection complicated with lung injury through mediating the release of MMP9 from macrophages. Am J Transl Res. 2016;8(3):1426-36. PMID 27186269.

3. Pan X, Lu J, Cheng W, Yang Y, Zhu J, Jin M. Independent factors related to preoperative acute lung injury in 130 adults undergoing Stanford type-A acute aortic dissection surgery: a single-center cross-sectional clinical study. J Thorac Dis. 2018;10(7):4413-23. https://doi.org/10.21037/ jtd.2018.06.140.

4. Elsayed RS, Cohen RG, Fleischman F, Bowdish ME. Acute type A aortic dissection. Cardiol Clin. 2017;35(3):331-45. https:// doi.org/10.1016/j.ccl.2017.03.004.

5. Han S, Mallampalli RK. The acute respiratory distress syndrome: from mechanism to translation. J Immunol. 2015;194(3):85560. https://doi.org/10.4049/jimmunol.1402513.

6. Wu D, Shen YH, Russell L, Coselli JS, LeMaire SA. Molecular mechanisms of thoracic aortic dissection. J Surg Res. 2013;184(2):907-24. https://doi.org/ 10.1016/j. jss.2013.06.007.

7. Huebener P, Pradere JP, Hernandez C, Gwak GY, Caviglia $J M, M u X$, et al. The HMGB1/RAGE axis triggers neutrophilmediated injury amplification following necrosis. J Clin Invest. 2015;125(2):539-50. https://doi.org/10.1172/JCI76887.

8. El Gazzar M. HMGB1 modulates inflammatory responses in LPS-activated macrophages. Inflamm Res. 2007;56(4):162-7. https://doi.org/ 10.1007/s00011-006-6112-0.
9. Hagiwara S, Iwasaka H, Matsumoto S, Noguchi T. Changes in cell culture temperature alter release of inflammatory mediators in murine macrophagic RAW264.7 cells. Inflamm Res. 2007;56(7):297303. https://doi.org/ 10.1007/s00011-007-6161-z.

10. Qin C, Gu J, Qian H, Liu R, Xu F, Li Y, et al. Potential mechanism of post-acute aortic dissection inflammatory responses: the role of mtDNA from activated platelets. Cardiology. 2016;135(4):22835. https://doi.org/10.1159/000446870.

11. Luo F, Zhou XL, Li JJ, Hui RT. Inflammatory response is associated with aortic dissection. Ageing Res Rev. 2009;8(1):31-5. https:// doi.org/10.1016/j.arr.2008.08.001.

12. Wang $Y$, Xue S, Zhu H. Risk factors for postoperative hypoxemia in patients undergoing Stanford A aortic dissection surgery. J Cardiothorac Surg. 2013;8:118. https://doi.org/10.1186/17498090-8-118.

13. $Y u$ Y, Yang $L, L v J$, Huang $X, Y i J$, Pei $C$, et al. The role of high mobility group box 1 (HMGB-1) in the diabetic retinopathy inflammation and apoptosis. Int J Clin Exp Pathol. 2015;8(6):6807-13. PMID 26261566.

14. Andersson $\mathrm{U}$, Yang $\mathrm{H}$, Harris $\mathrm{H}$. High-mobility group box 1 protein (HMGB1) operates as an alarmin outside as well as inside cells. Semin Immunol. 2018;38:40-8. https://doi.org/ 10.1016/j.smim.2018.02.011.

15. Shi Y, Zhang L, Teng J, Miao W. HMGB1 mediates microglia activation via the TLR4/NF- $\kappa B$ pathway in coriaria lactone induced epilepsy. Mol Med Rep. 2018;17(4):5125-31. https:// doi.org/10.3892/mmr.2018.8485.

16. Zhou H, Wang X, Zhang B. Depression of IncRNANEAT1 antagonizes LPS-evoked acute injury and inflammatory response in alveolar epithelial cells via HMGB1-RAGE Signaling. Mediators Inflamm. 2020;2020:8019467. https://doi.org/10.1155/2020/8019467. 


\section{ERRATUM}

https://doi.org/10.1590/1806-9282.20210395ERRATUM

In the manuscript "Associations of high-mobility group box 1 and receptor for advanced glycation end products with acute lung injury in patient with acute aortic dissection", https://doi.org/10.1590/1806-9282.20210395, published in the Rev Assoc Med Bras. 2021;67(9):1251-1255, on page 1255:

Where it reads:

\section{AUTHORS" CONTRIBUTIONS}

JY: Conceptualization, Data curation, Formal analysis. ZZ: Funding acquisition, Investigation. KZ: Methodology, Project administration. JC: Supervision, Validation, Visualization. HW: Writing-original draft.

It should read:

\section{AUTHORS" CONTRIBUTIONS}

JY: Conceptualization, Data curation, Formal analysis. ZZ: Funding acquisition, Investigation. KZ: Methodology, Project administration. JC: Supervision, Validation, Visualization. HW: Writing-original draft. ZZ and KZ: Contributed equally. 